\title{
Intrinsic five-quark Fock states of baryons and meson-nucleon $\sigma$-terms
}

\author{
C. S. $A n^{* i}$ \\ Institute of High Energy Physics, CAS, P. O. Box 918-4, Beijing 100049, China \\ Theoretical Physics Center for Science Facilities, CAS, P. O. Box 918-4, Beijing 100049, China \\ E-mail: ancs@ihep.ac.cn \\ B. Saghai \\ Institut de Recherche sur les lois Fondamentales de l'Univers, DSM/Irfu, CEA/Saclay, F-91191 \\ Gif-sur-Yvette, France \\ E-mail: bijan.saghai@cea.fr
}

\begin{abstract}
An extended chiral constituent quark approach, which embodies higher Fock five-quark components in the baryons wave-functions, is employed to study the sea quark content of the octet baryons. We report on the probabilities of $\bar{u}, \bar{d}$ and $\bar{s}$ in the nucleon, $\Lambda, \Sigma$ and $\Xi$ baryons, arising from the intrinsic five-quark states in the baryons wave functions. Based on those probabilities, results for meson-nucleon $\sigma$-terms; namely $\sigma_{\pi N}, \sigma_{K N}$ and $\sigma_{\eta N}$, are presented.
\end{abstract}

Sixth International Conference on Quarks and Nuclear Physics,

April 16-20, 2012

Ecole Polytechnique, Palaiseau, Paris

\footnotetext{
* Speaker.

${ }^{\dagger}$ It is a pleasure to thank the Organizers for partial financial support to attend the Conference.
} 


\section{Introduction}

The pion-nucleon nucleon sigma term, $\sigma_{\pi N}$, can be considered as a test of QCD, where the chiral-symmetry breaking is due to representation of $S U(3) \times S U(3)$; see e.g. Refs. $[1,2,3,4]$ and references therein.

A thorough investigation of baryon's structure involves nonpertubative QCD. Moreover, the strange quark component, a purely vacuum polarization effects, plays a crucial role in that realm. In a series of recent papers $[5,6,7]$ we have studied contributions from the genuine five-quark components in baryons to various properties of baryons [5, 6] and have determined [7] the probabilities of five-quark components $(u \bar{u}, d \bar{d}$ and $s \bar{s})$ in ground state octet baryons. In this paper we extend our studies to the meson-nucleon $\sigma$-terms.

Our formalism is based on an extended chiral constituent quark model ( $E \chi C Q M)$ approach and embodies all possible five-quark mixtures in the octet baryons wave-functions. Using our approach, we put forward predictions on the probabilities of $u \bar{u}, d \bar{d}$ and $s \bar{s}$ in ground state baryons.

The present manuscript is organized in the following way: in section 2, we briefly present the theoretical frame. Numerical results are given in section 3, putting forward predictions for the probabilities of different five-quark configurations in the nucleon, $\Lambda, \Sigma$ and $\Xi$ baryons and extract the meson-nucleon $\sigma$-terms. Finally, section 4 contains summary and conclusions.

\section{Theoretical frame}

In the extended constituent quark model [7], wave function for a baryon is expressed as

$$
|\psi\rangle_{B}=\frac{1}{\sqrt{\mathscr{N}}}\left[|Q Q Q\rangle+\sum_{i, n_{r}, l} C_{i n_{r} l}\left|Q Q Q(Q \bar{Q}), i, n_{r}, l\right\rangle\right]
$$

where the first term is the conventional wave function for the baryon with three constituent quarks, and the second term is a sum over all possible higher Fock components with a $Q \bar{Q}$ pair. Here we denote light quark-antiquark pair as $Q \bar{Q} \equiv q \bar{q}$ (with $q \equiv u, d$,) and strange quark-antiquark pairs as $Q \bar{Q} \equiv s \bar{s}$. Different possible orbital-flavor-spin-color configurations of the four-quark subsystems in the five-quark system are numbered by $i ; n_{r}$ and $l$ denote the inner radial and orbital quantum numbers, respectively. The coefficients $C_{i n_{r} l}$ in Eq. (2.1) can be related to the coupling between the valence three-quark and the corresponding five-quark components.

$$
C_{i n_{r} l}=\frac{\left\langle Q Q Q(Q \bar{Q}), i, n_{r}, l|\hat{T}| Q Q Q\right\rangle}{M_{B}-E_{i n_{r} l}}
$$

where $M_{B}$ is the mass of baryon $\mathrm{B}, E_{i n_{r} l}$ the energy of the relevant five-quark component, and $\hat{T}$ a model dependent coupling operator.

The matrix elements of $\hat{T}$ between the three- and five-quark configurations are derived using $\mathrm{a}^{3} P_{0}$ version for the transition coupling operator

$$
\hat{T}=-\gamma \sum_{j} \mathscr{F}_{j, 5}^{00} \mathscr{C}_{j, 5}^{00} C_{O F S C}\left[\sum_{m}\langle 1, m ; 1,-m \mid 00\rangle \chi_{j, 5}^{1, m} \mathscr{Y}_{j, 5}^{1,-m}\left(\vec{p}_{j}-\vec{p}_{5}\right) b^{\dagger}\left(\vec{p}_{j}\right) d^{\dagger}\left(\vec{p}_{5}\right)\right],
$$


where $\gamma$ is a dimensionless constant of the model, $\mathscr{F}_{i, 5}^{00}$ and $\mathscr{C}_{i, 5}^{00}$ denote the flavor and color singlet of the quark-antiquark pair $Q_{i} \bar{Q}$ in the five-quark system, and $C_{O F S C}$ is an operator to calculate the orbital-flavor-spin-color overlap between the residual three-quark configuration in the five-quark system and the valence three quark system.

The derived matrix elements $T$ for the 34 five-quark configurations show that only 17 configurations, corresponding to the orbital quantum number $l=1$ and radial quantum number $n_{r}=0$, survive and matrix elements $T$ for all other ones vanish.

The probability of the sea quark in each baryon $B$ reads

$$
P_{B}^{Q \bar{Q}}=\frac{1}{\mathscr{N}} \sum_{i=1}^{17}\left[\left(\frac{T_{i}^{Q \bar{Q}}}{M_{B}-E_{i}^{Q \bar{Q}}}\right)^{2}\right]
$$

with the normalization factor

$$
\mathscr{N} \equiv 1+\sum_{i=1}^{17} \mathscr{N}_{i}=1+\sum_{i=1}^{17}\left[\left(\frac{T_{i}^{u \bar{u}}}{M_{B}-E_{i}^{u \bar{u}}}\right)^{2}+\left(\frac{T_{i}^{d \bar{d}}}{M_{B}-E_{i}^{d \bar{d}}}\right)^{2}+\left(\frac{T_{i}^{s \bar{s}}}{M_{B}-E_{i}^{s \bar{s}}}\right)^{2}\right] .
$$

Notice that in Eq. (2.5) the first term is due to the valence three-quark states, while the second term comes from the five-quark mixtures.

Sigma terms are in general expressed via the strangeness fraction $y_{N}$ and the non-singlet component $\hat{\sigma}$ defined as

$$
\begin{aligned}
y_{N} & =\frac{2\langle p|s \bar{s}| p\rangle}{\langle p|u \bar{u}+d \bar{d}| p\rangle}=\frac{2 P_{s \bar{s}}}{3+2\left(P_{u \bar{u}}+P_{d \bar{d}}\right)}, \\
\hat{\sigma} & =\hat{m}\langle p|u \bar{u}+d \bar{d}-2 s \bar{s}| p\rangle .
\end{aligned}
$$

Then, the $\sigma$-terms read as follows:

$$
\begin{aligned}
\sigma_{\pi N} & =\frac{\hat{\sigma}}{1-2 y_{N}}, \\
\sigma_{K N}^{I=0} & =\frac{\hat{m}+m_{s}}{4 \hat{m}}\left(1+2 y_{N}\right) \sigma_{\pi N}, \\
\sigma_{\eta N} & =\frac{\hat{m}+2 y_{N} m_{s}}{3 \hat{m}} \sigma_{\pi N},
\end{aligned}
$$

where $\hat{m}=\left(m_{u}+m_{d}\right) / 2$, with $m_{u}, m_{d}$ and $m_{s}$ current quark masses.

\section{Results and discussion}

In Table (1) our results for probabilities (Eq. 2.4) of $q \bar{q}(q \equiv u, d)$ and $s \bar{s}$ are given for each of the 17, non-vanishing, five-quark configurations. Then, predictions of our model for the sea content of the octet baryons, in particle basis, are reported in Table (2). Notice small discrepancies between the values in the above Tables and those in Tables (VI) and (VII) in our recent paper [7], due to a misprint with respect to the $\mathrm{i}=12$ configuration numbers. The only significant change concrns the $d \bar{d}$ component in $\Xi^{\circ}$, the probability of which increases from $12.1 \%$ to $13.8 \%$.

Finally, using Eqs. (2.6)-(2.8), we extract values for the meson-nucleon $\sigma$-terms; namely, $\sigma_{\pi N}, \sigma_{K N}$ and $\sigma_{\eta N}$ (Table (3)). 
Table 1: Predictions for probabilities of different five-quark components in the nucleon, $\Lambda, \Sigma$ and $\Xi$. Upper and lower panels are for the configurations with $L_{4 q}=1$ and $L_{4 q}=0$, respectively.

\begin{tabular}{|c|c|c|c|c|c|c|}
\hline $\mathrm{N}^{\circ}$ & Configuration & Sea flavor & $\mathrm{N}$ & $\Lambda$ & $\Sigma$ & $\Xi$ \\
\hline \multirow[t]{2}{*}{1} & {$[4]_{F S}[22]_{F}[22]_{S}$} & $q \bar{q}$ & 0.146 & 0.115 & 0.066 & 0.081 \\
\hline & & $s \bar{s}$ & 0.010 & 0 & 0.020 & 0 \\
\hline \multirow[t]{2}{*}{2} & {$[4]_{F S}[31]_{F}^{1}[31]_{S}$} & $q \bar{q}$ & 0.073 & 0 & 0.054 & 0.028 \\
\hline & & $s \bar{S}$ & 0 & 0 & 0.009 & 0.016 \\
\hline \multirow[t]{2}{*}{3} & {$[4]_{F S}[31]_{F}^{2}[31]_{S}$} & $q \bar{q}$ & 0 & 0.052 & 0.003 & 0.006 \\
\hline & & $s \bar{S}$ & 0.006 & 0.013 & 0 & 0 \\
\hline \multirow[t]{2}{*}{4} & {$[31]_{F S}[211]_{F}[22]_{S}$} & $q \bar{q}$ & 0 & 0.003 & 0.011 & 0.010 \\
\hline & & $s \bar{S}$ & 0.004 & 0.003 & 0 & 0 \\
\hline \multirow[t]{2}{*}{5} & {$[31]_{F S}[211]_{F}[31]_{S}$} & $q \bar{q}$ & 0 & 0.002 & 0.009 & 0.008 \\
\hline & & $s \bar{s}$ & 0.003 & 0.002 & 0 & 0 \\
\hline \multirow[t]{2}{*}{6} & {$[31]_{F S}[22]_{F}[31]_{S}$} & $q \bar{q}$ & 0.007 & 0.011 & 0.004 & 0.012 \\
\hline & & $s \bar{S}$ & 0.002 & 0 & 0.005 & 0 \\
\hline \multirow[t]{2}{*}{7} & {$[31]_{F S}[31]_{F}^{1}[22]_{S}$} & $q \bar{q}$ & 0.018 & 0 & 0.018 & 0.011 \\
\hline & & $s \bar{s}$ & 0 & 0 & 0.004 & 0.009 \\
\hline \multirow[t]{2}{*}{8} & {$[31]_{F S}[31]_{F}^{2}[22]_{S}$} & $q \bar{q}$ & 0 & 0.016 & 0.001 & 0.002 \\
\hline & & $s \bar{s}$ & 0.003 & 0.006 & 0 & 0 \\
\hline \multirow[t]{2}{*}{9} & {$[31]_{F S}[31]_{F}^{1}[31]_{S}$} & $q \bar{q}$ & 0.005 & 0 & 0.006 & 0.003 \\
\hline & & $s \bar{S}$ & 0 & 0 & 0.001 & 0.003 \\
\hline \multirow[t]{2}{*}{10} & {$[31]_{F S}[31]_{F}^{2}[31]_{S}$} & $q \bar{q}$ & 0 & 0.004 & 0 & 0.001 \\
\hline & & $s \bar{s}$ & 0.001 & 0.002 & 0 & 0 \\
\hline \multirow[t]{2}{*}{11} & {$[31]_{F S}[211]_{F}[22]_{S}$} & $q \bar{q}$ & 0 & 0.006 & 0.022 & 0.021 \\
\hline & & $s \bar{s}$ & 0.009 & 0.006 & 0 & 0 \\
\hline \multirow[t]{2}{*}{12} & {$[31]_{F S}[211]_{F}[31]_{S}$} & $q \bar{q}$ & 0 & 0.005 & 0.019 & 0.018 \\
\hline & & $s \bar{S}$ & 0.008 & 0.006 & 0 & 0 \\
\hline \multirow[t]{2}{*}{13} & {$[31]_{F S}[22]_{F}[31]_{S}$} & $q \bar{q}$ & 0.015 & 0.025 & 0.010 & 0.028 \\
\hline & & $s \bar{S}$ & 0.004 & 0 & 0.011 & 0 \\
\hline \multirow[t]{2}{*}{14} & {$[31]_{F S}[31]_{F}^{1}[22]_{S}$} & $q \bar{q}$ & 0.042 & 0 & 0.043 & 0.026 \\
\hline & & $s \bar{S}$ & 0 & 0 & 0.010 & 0.022 \\
\hline \multirow[t]{2}{*}{15} & {$[31]_{F S}[31]_{F}^{2}[22]_{S}$} & $q \bar{q}$ & 0 & 0.037 & 0.002 & 0.005 \\
\hline & & $s \bar{S}$ & 0.007 & 0.015 & 0 & 0 \\
\hline \multirow[t]{2}{*}{16} & {$[31]_{F S}[31]_{F}^{1}[31]_{S}$} & $q \bar{q}$ & 0.012 & 0 & 0.013 & 0.008 \\
\hline & & $s \bar{s}$ & 0 & 0 & 0.003 & 0.007 \\
\hline \multirow[t]{2}{*}{17} & {$[31]_{F S}[31]_{F}^{2}[31]_{S}$} & $q \bar{q}$ & 0 & 0.010 & 0 & 0.001 \\
\hline & & $s \bar{S}$ & 0.002 & 0.004 & 0 & 0 \\
\hline
\end{tabular}

The pion-nucleon $\sigma$-term is the most studied one and the often quoted value was reported in Ref. [8]: $\sigma_{\pi N}=45 \pm 8 \mathrm{MeV}$, which was obtained from $\pi N$ data analysis with taking into account the 
Table 2: Predictions for the sea content of the octet baryons.

\begin{tabular}{lcccc}
\hline \hline Baryon & $\bar{u}$ & $\bar{d}$ & $\bar{s}$ & $\bar{u}+\bar{d}+\bar{s}$ \\
\hline $\mathrm{p}$ & 0.100 & 0.218 & 0.058 & 0.376 \\
$\Lambda$ & 0.143 & 0.143 & 0.058 & 0.344 \\
$\Sigma^{+}$ & 0.101 & 0.179 & 0.063 & 0.343 \\
$\Sigma^{0}$ & 0.140 & 0.140 & 0.063 & 0.343 \\
$\Xi^{0}$ & 0.131 & 0.138 & 0.057 & 0.326 \\
\hline \hline
\end{tabular}

current algebra result generated by the quark masses. As it can be inferred from Table (3), various results reported in literature agree with the canonical value within $2 \sigma$, and our result falls at the lower band within $1 \sigma$.

In Table (3) results from other authors are also shown, coming from Chiral Constituent Quark Models $(\chi C Q M)$ [10], Perturbative Chiral Constituent Quark (P $\chi C Q)$ [11], Lattice QCD (LQCD) [2, 3 , 4], and Chiral Perturbation Theory $(\chi P T)[12]$.

Table 3: Predictions for the meson-nucleon $\sigma$-terms (in MeV), with $\hat{\sigma}=35 \mathrm{MeV}$ (Ref. [8]) and $m_{s} / \hat{m}=25$ (Ref. [9]).

\begin{tabular}{llccc}
\hline \hline Reference & Approach & $\sigma_{\pi N}$ & $\sigma_{K N}$ & $\sigma_{\eta N}$ \\
\hline Present work & $E \chi C Q M$ & 37 & 256 & 32 \\
Dahiya et al. [10] & $\chi C Q M$ & 31 & 196 & 31 \\
Inoue et al. [11] & $P \chi C Q$ & 55 & 33 & 96 \\
Durr et al. [2] & LQCD & $39 \pm 4$ & & \\
Bali et al. [3] & LQCD & $38 \pm 12$ & & \\
Shanahan et al. [4] & LQCD & $45 \pm 6$ & $300 \pm 40$ & \\
Alarcon et al. [12] & $\chi P T$ & $43 \pm 5$ & & \\
Alarcon et al. [12] & $\chi P T$ & $59 \pm 7$ & & \\
\hline \hline
\end{tabular}

Our result for $\sigma_{\pi N}$ is close enough to all other predictions, except in the case of a recent $\chi P T$ approach [12], where two different partial wave analysis (PWA) lead to: $43 \pm 5 \mathrm{MeV}$ and $59 \pm 7$ $\mathrm{MeV}$; only the former being compatible with ours. It is worth noting that the smaller value is obtained using the PWA of the Karlsruhe-Helsinki group [13] and the larger one via a more recent PWA provided by SAID [14].

In Ref. [10] it is reported that non-relativistic quark model underestimates all three mesonnucleon $\sigma$-terms, and $\chi C Q M$ under exact $S U(3)$ symmetry overestimates them. Actually, taking into account the $S U(3)$ symmetry breaking, results come out very close to those found within the present work. That is also the case for the extracted $\sigma_{K N}$ and $\sigma_{\eta N}$.

Results from a $P \chi C Q$ [11] lead to $\sigma$-terms, all three in disagreement with our findings.

For $\sigma_{K N}$, our result is compatible with those reported in Refs. [4, 10].

Finally, lattice QCD calculations $[2,3,4]$ are producing results mainly for $\sigma_{\pi N}$, with including 
strangeness content of the baryons and find strange $\sigma$-term to be in the range $20-50 \mathrm{MeV}$, though with rather large uncertainties [4]. For $\sigma_{\pi N}, L Q C D$ approaches favor values significantly smaller than those obtained via dispersion relations, using the SAID $\pi N$ phase-shift analysis [15], and giving [16] $\sigma_{\pi N}=81 \pm 6$.

\section{Summary and conclusions}

An extended chiral constituent quark model, embodying genuine five-quark mixture in the ground state baryon octet wave functions, was briefly presented, underlining the sea quark content. We put forward predictions of our complete model for the percentage, per flavor, of the sea quark content for $N, \Lambda, \Sigma$ and $\Xi$.

Those predictions were then used to calculate the meson-nucleon $\sigma$-terms and led to: $\sigma_{\pi N}=37$ $\mathrm{MeV}, \sigma_{K N}=256 \mathrm{MeV}$ and $\sigma_{\eta N}=32 \mathrm{MeV}$. Our findings compare satisfactorily with results from recent studies based on chiral constituent quark model [10] and lattice QCD [2, 3, 4], while showing significant discrepancies with values determined via perturbative chiral quark approach [11] and dispersion relations [16].

Extension of the present work to predict $\sigma$-terms for meson- $\Lambda$, meson- $\Sigma$ and meson- $\Xi$ is in progress.

\section{References}

[1] F. Huang, F. G. Cao and B. Q. Ma, Phys. Rev. D 76, 114016 (2007) [arXiv:0711.0675 [hep-ph]].

[2] S. Durr et al., Phys. Rev. D 85, 014509 (2012) [arXiv:1109.4265 [hep-lat]].

[3] G. S. Bali et al. [QCDSF Collaboration], Phys. Rev. D 85, 054502 (2012) [arXiv:1111.1600 [hep-lat]].

[4] P. E. Shanahan, A. W. Thomas and R. D. Young, arXiv:1205.5365 [nucl-th].

[5] C. S. An, B. Saghai, S. G. Yuan and J. He, Phys. Rev. C 81, 045203 (2010) [arXiv:1002.4085 [nucl-th]].

[6] C. S. An and B. Saghai, Phys. Rev. C 84, 045204 (2011) [arXiv:1108.3282 [nucl-th]].

[7] C. S. An and B. Saghai, Phys. Rev. C 85, 055203 (2012) [arXiv:1204.0300 [hep-ph]].

[8] J. Gasser, H. Leutwyler and M. E. Sainio, Phys. Lett. B 253, 252 (1991).

[9] H. Leutwyler, Phys. Lett. B 378, 313 (1996) [hep-ph/9602366].

[10] H. Dahiya and N. Sharma, AIP Conf. Proc. 1388, 439 (2011) [arXiv:1107.3927 [hep-ph]].

[11] T. Inoue, V. E. Lyubovitskij, T. Gutsche and A. Faessler, Phys. Rev. C 69, 035207 (2004) [hep-ph/0311275].

[12] J. M. Alarcon, J. Martin Camalich and J. A. Oller, Phys. Rev. D 85, 051503 (2012) [arXiv:1110.3797 [hep-ph]].

[13] R. Koch, Z. Phys. C 15, 161 (1982).

[14] R. A. Arndt, W. J. Briscoe, I. I. Strakovsky and R. L. Workman, Phys. Rev. C 74, 045205 (2006) [nucl-th/0605082].

[15] R. A. Arndt, W. J. Briscoe, I. I. Strakovsky, R. L. Workman and M. M. Pavan, Phys. Rev. C 69, 035213 (2004) [nucl-th/0311089].

[16] G. E. Hite, W. B. Kaufmann and R. J. Jacob, Phys. Rev. C 71, 065201 (2005). 